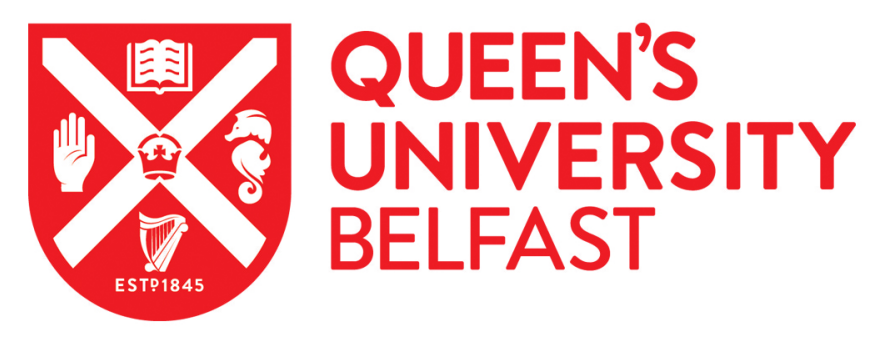

\title{
Polarization Dependence of Bulk lon Acceleration from Ultrathin Foils Irradiated by High-Intensity Ultrashort Laser Pulses
}

Scullion, C., Doria, D., Romagnani, L., Sgattoni, A., Naughton, K., Symes, D. R., McKenna, P., Macchi, A., Zepf, M., Kar, S., \& Borghesi, M. (2017). Polarization Dependence of Bulk Ion Acceleration from Ultrathin Foils Irradiated by High-Intensity Ultrashort Laser Pulses. Physical Review Letters, 119(5), [054801]. https://doi.org/10.1103/PhysRevLett.119.054801

Published in:

Physical Review Letters

Document Version:

Publisher's PDF, also known as Version of record

Queen's University Belfast - Research Portal:

Link to publication record in Queen's University Belfast Research Portal

Publisher rights

(C) 2017 American Physical Society.

This work is made available online in accordance with the publisher's policies. Please refer to any applicable terms of use of the publisher.

\section{General rights}

Copyright for the publications made accessible via the Queen's University Belfast Research Portal is retained by the author(s) and / or other copyright owners and it is a condition of accessing these publications that users recognise and abide by the legal requirements associated with these rights.

Take down policy

The Research Portal is Queen's institutional repository that provides access to Queen's research output. Every effort has been made to ensure that content in the Research Portal does not infringe any person's rights, or applicable UK laws. If you discover content in the Research Portal that you believe breaches copyright or violates any law, please contact openaccess@qub.ac.uk. 


\title{
Polarization Dependence of Bulk Ion Acceleration from Ultrathin Foils Irradiated by High-Intensity Ultrashort Laser Pulses
}

\author{
C. Scullion, ${ }^{1}$ D. Doria, ${ }^{1, *}$ L. Romagnani,${ }^{2}$ A. Sgattoni,${ }^{3, \dagger}$ K. Naughton, ${ }^{1}$ D. R. Symes, ${ }^{4}$ P. McKenna, ${ }^{5}$ \\ A. Macchi, ${ }^{3,6}$ M. Zepf, ${ }^{1,7}$ S. Kar, ${ }^{1}$ and M. Borghesi ${ }^{1, \$}$ \\ ${ }^{1}$ Centre for Plasma Physics, School of Mathematics and Physics, Queen's University Belfast, Belfast BT7 INN, United Kingdom \\ ${ }^{2}$ LULI, École Polytechnique, CNRS, Route de Saclay, 91128 Palaiseau Cedex, France \\ ${ }^{3}$ Istituto Nazionale di Ottica, Consiglio Nazionale delle Ricerche (CNR/INO), Laboratorio Adriano Gozzini, 56124 Pisa, Italy \\ ${ }^{4}$ Central Laser Facility, Rutherford Appleton Laboratory, Oxfordshire OX11 OQX, United Kingdom \\ ${ }^{5}$ SUPA, Department of Physics, University of Strathclyde, Glasgow G4 ONG, United Kingdom \\ ${ }^{6}$ Dipartimento di Fisica Enrico Fermi, Università di Pisa, 56127 Pisa, Italy \\ ${ }^{7}$ Helmholtz Institute Jena, 07743 Jena, Germany \\ (Received 23 May 2016; published 2 August 2017)
}

\begin{abstract}
The acceleration of ions from ultrathin $(10-100 \mathrm{~nm})$ carbon foils has been investigated using intense $\left(\sim 6 \times 10^{20} \mathrm{~W} \mathrm{~cm}^{-2}\right)$ ultrashort (45 fs) laser pulses, highlighting a strong dependence of the ion beam parameters on the laser polarization, with circularly polarized $(\mathrm{CP})$ pulses producing the highest energies for both protons and carbons $(25-30 \mathrm{MeV} /$ nucleon); in particular, carbon ion energies obtained employing CP pulses were significantly higher ( 2.5 times) than for irradiations employing linearly polarized pulses. Particle-in-cell simulations indicate that radiation pressure acceleration becomes the dominant mechanism for the thinnest targets and CP pulses.
\end{abstract}

DOI: 10.1103/PhysRevLett.119.054801

Laser-driven ion acceleration is an area of research attracting considerable interest $[1,2]$ due to a number of attractive features, such as the compactness of the approach compared to conventional accelerators, the excellent transverse [3] and longitudinal [4] emittance of the beams, and the prospect of achieving high ion energies (100s of $\mathrm{MeV} /$ nucleon) with next-generation laser sources [5]. Research in this area is also motivated by applicative prospects in fields ranging from high-energy-density physics [6-8] to biology and medicine [9-11], neutron production [12,13], and nuclear physics [14,15].

Until recently, most experimental research has been devoted to proton acceleration from laser-irradiated foils with thicknesses in the $\mu \mathrm{m}$ range, with results interpreted in terms of the target normal sheath acceleration (TNSA) mechanism [16], where ions are accelerated by space charge fields generated at the rear surface of the target by relativistic electrons. Lately, attention has shifted to a number of different volumetric acceleration mechanisms [1] displaying promising features in view of the aforementioned applications. Some of these novel mechanisms are ideally implemented by employing low-mass targets, which has motivated recent experimental research using ultrathin foils, with thicknesses in the nm range [17-20]. In particular, ion acceleration harnessing the extreme radiation pressure exerted by intense lasers has been highlighted as a particularly promising approach to attain energies in the 100s of MeV/u range and above, via the so-called light sail (LS) radiation pressure acceleration (RPA) mechanism [21-26]. Controlling the polarization of the laser pulse has been proposed as a means of preserving target opacity during the laser irradiation and to achieve an efficient radiation pressure drive on ultrathin foils. A linearly polarized (LP) laser pulse incident normally on to a flat foil drives, through the $2 \omega$ component of the $\mathbf{J} \times \mathbf{B}$ force, a sweeping oscillation of the density profile and causes strong absorption and hot electron generation; however, in the case of circular polarization (CP) at normal incidence, the oscillatory force component normal to the target vanishes, suppressing hot electron production. The use of CP laser pulses should, therefore, facilitate entering regimes where RPA is the dominant acceleration mechanism for ultrathin foils, since the reduced heating of the electrons of the foil delays the onset of target transparency during laser irradiation [22-26].

Accessing efficient RPA acceleration from ultrathin foils relies heavily on many critical parameters. In particular, while a quasi-1D drive is proven to be highly effective in numerical investigations, the finite spot-size effects associated with tight focusing (which are unavoidable in current experiments due to pulse energy limitations, particularly with femtosecond systems) have been highlighted (e.g., in [27]) as a major obstacle to the implementation of LS acceleration. Nevertheless, some experiments employing tens of fs $\mathrm{CP}$ pulses, focused at mid- $10^{19} \mathrm{~W} \mathrm{~cm}^{-2}$ intensities on nm-scale foils [28,29], reported features in the proton and $\mathrm{C}^{6+}$ spectra (at $\sim \mathrm{MeV}$ energies) which could be interpreted in terms of LS-RPA acceleration. Henig et al. [28], in particular, showed the emergence of a broad peak feature in the carbon spectrum when using $\mathrm{CP}$ pulses, 
although at lower ion energies than observed in LP shots. In recent experiments employing ultrashort pulses, a polarization-dependent enhancement in ion acceleration efficiency was induced by laser-pulse self-focusing in lowdensity media preceding the foil [30]. In longer pulse (ps) regimes, Kar et al. [31] reported ion acceleration in a hybrid RPA-TNSA mode that displayed some of the characteristic features of LS RPA (e.g., the fast scaling), but did not highlight a significant polarization dependence; this was likely due to the effect of target deformation within the relatively long interaction duration, which results in increasingly oblique incidence away from the center of the focal spot.

This Letter reports on the first clear evidence of the polarization-dependent dominance of RPA-LS in accelerating ions during the interaction of ultrashort (45 fs) laser pulses with ultrathin carbon foils. The effect is evident from measuring the spectra of the carbon ions, where significantly higher (more than twice) energies were obtained employing CP laser pulses compared to irradiations with LP pulses. Multidimensional particle-in-cell (PIC) simulations reproduce the salient features of the data and indicate that two different acceleration processes are dominant for CP and LP laser pulses at the thinnest targets employed. While an efficient LS drive is sustained for most of the pulse in $\mathrm{CP}$, for LP an earlier transition to transparency and stronger electron heating leads to a lessefficient acceleration.

The experiment was carried out on the GEMINI Ti: sapphire laser system at the Rutherford Appleton Laboratory, STFC, United Kingdom. The laser delivered $\sim 6 \mathrm{~J}$ energy on target in pulses of 800-nm wavelength $(\lambda)$ and 45 -fs full width at half maximum (FWHM) duration $(\tau)$, after being reflected off a double-plasma-mirror (DPM) [32] arrangement. The recollimated laser beam after the plasma mirrors was focused on the targets at normal incidence by an $f / 2$ off-axis parabolic mirror (OAP), delivering peak intensities on target $\sim 6 \times 10^{20} \mathrm{~W} \mathrm{~cm}^{-2}$. The laser polarization on the target was varied from LP to CP by employing a zero-order quarter wave plate (WP), placed between the plasma mirror and the focusing parabola (see Fig. 1). Amorphous carbon targets of thickness $L$ in the $10-100 \mathrm{~nm}$ range were irradiated. The energy spectra of the ions accelerated from the interaction were diagnosed, by a Thomson parabola spectrometer (TPS) with BAS-TR image plate (IP) detectors [33,34], along the laser axis (also target normal axis) with an acceptance angle of $1.1 \mu \mathrm{Sr}$. The energy-resolved spatial profiles of the ion beam were recorded by using stacks of radiochromic films (RCFs) and CR-39 detectors. Two types of RCF were used in the stack, HD-V2 and EBT2, as their different sensitivities allowed us to diagnose the proton beam over the large differences in particle density present across the spectrum.

Figure 2(a) shows the IP scans for typical CP and LP shots with target thickness $10 \mathrm{~nm}$. The proton and $\mathrm{C}^{6+}$

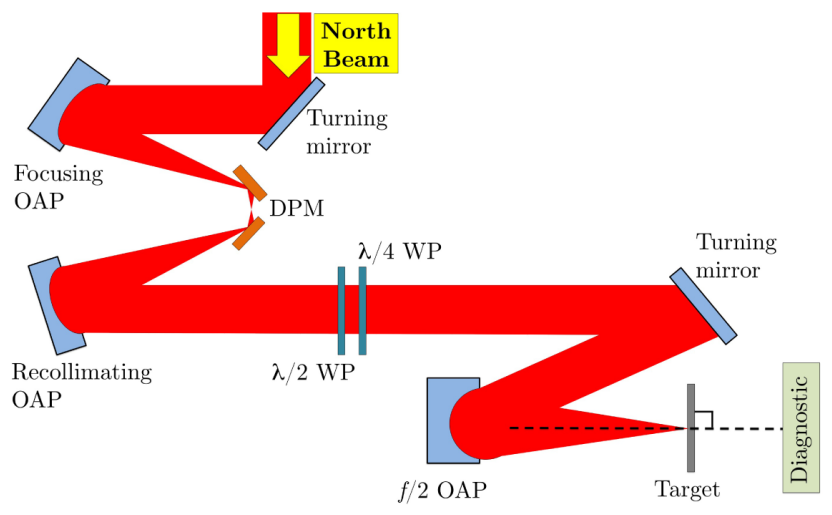

FIG. 1. Schematic of the experimental setup. The laser was focused on the target by an $f / 2$ off-axis parabolic mirror, after being reflected off a DPM arrangement. The laser polarization on the target was varied from linear to circular by employing a zero-order quarter WP.

spectra obtained from these shots are compared in Figs. 2(b) and 2(c). As one can see for both protons and $\mathrm{C}^{6+}$ in Fig. 2(a), the traces extend significantly further for the $\mathrm{CP}$ case, indicating higher ion energies compared to the LP case. The difference is particularly striking for the carbon trace, where the maximum energies observed are higher by a factor of 2.5 . The spectrum is broad, with indication of a spectral bunch at the high-energy end of the spectrum. Spectral bunching at comparable, but slightly higher, energy per nucleon is seen in the proton spectrum.

Figure 3(a) shows the maximum energies $( \pm 1 \mathrm{MeV} / \mathrm{u})$ of $\mathrm{C}^{6+}$ ions obtained for each shot, plotted as a function of target thickness. The majority of points were obtained from the TPS; for $10-\mathrm{nm}$ targets, additional data points were
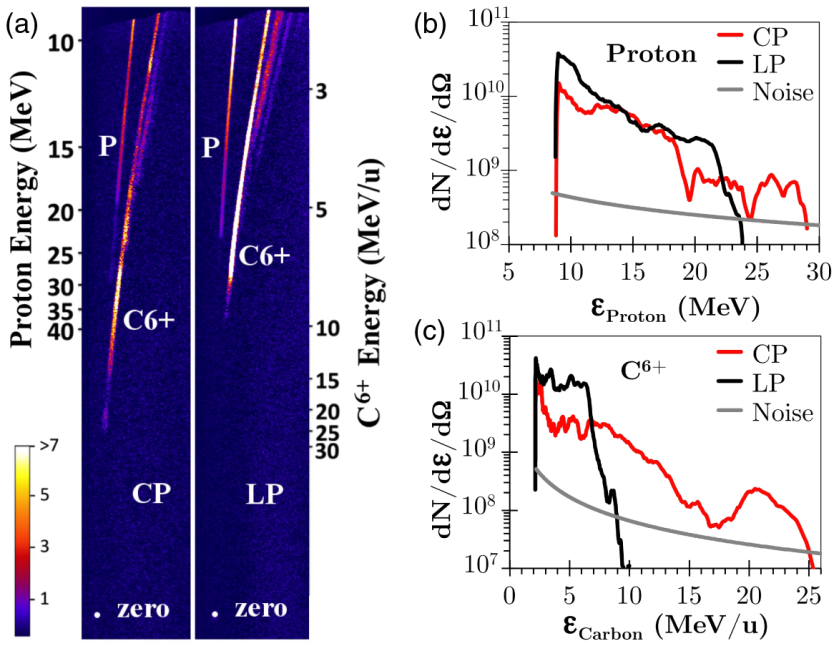

FIG. 2. (a) Raw data from BAS-TR image plates for CP and LP laser pulses irradiating 10-nm amorphous carbon targets. The corresponding CP (red) and LP (black) background-subtracted proton (b) and $\mathrm{C}^{6+}$ spectra (c) with vertical axis units of particles $/ \mathrm{MeV} / \mathrm{sr}$ are also shown. The noise level of $+2 \sigma$ is also plotted. 


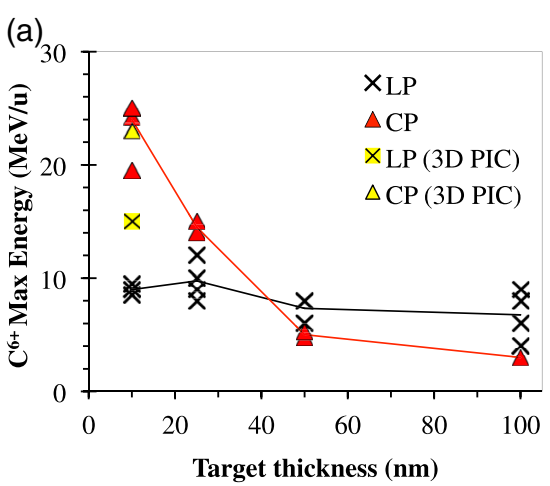

(b)

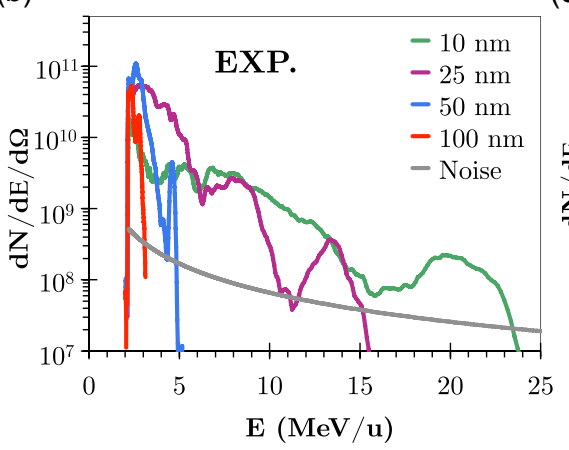

(c)

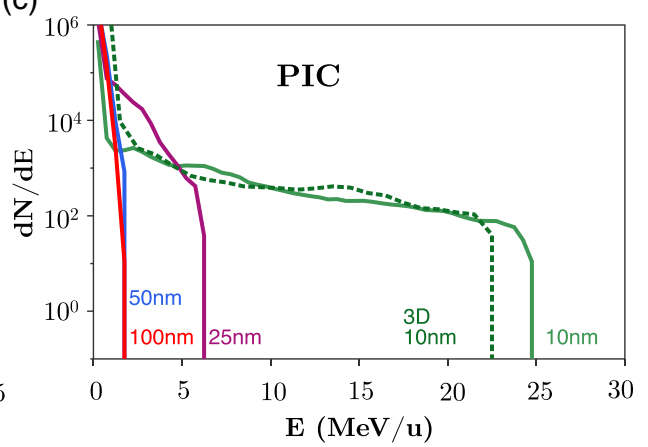

FIG. 3. (a) Maximum energy of $\mathrm{C}^{6+}$ ions vs target thickness for LP (black stars) and CP (red triangles). Data points for 10-nm amorphous carbon 3D PIC simulations are also shown (yellow). Note that solid red and black lines indicate average maximum energy for each thickness for CP and LP laser pulses, respectively, for clarity. (b) $\mathrm{C}^{6+}$ spectra from target thicknesses $10 \mathrm{~nm}$ (green), $25 \mathrm{~nm}$ (purple), $50 \mathrm{~nm}$ (blue), and $100 \mathrm{~nm}$ (red) with CP laser pulses (vertical axis units of particles/MeV/sr). (c) Spectra generated from PIC simulations: 2D (solid lines) and 3D (dotted line).

obtained from RCFs and CR-39. Representative $\mathrm{C}^{6+}$ spectra obtained for different target thicknesses and $\mathrm{CP}$ are plotted in Fig. 3(b). A sharp energy increase for decreasing target thickness is evident from the data, with a factor of 5 increase in energies when decreasing the thickness from 100 to $10 \mathrm{~nm}$.

For thick (50 and $100 \mathrm{~nm}$ ) targets, the maximum ion energies were higher for LP pulses. The ion energies for LP pulses increase only slightly for thinner targets, remaining at around $10 \mathrm{MeV} /$ nucleon for $\mathrm{C}^{6+}$ across the whole range of target thicknesses deployed in the experiment. On the other hand, when employing $\mathrm{CP}$ laser pulses and decreasing the thickness below 25-nm targets, the situation changes dramatically, producing significantly higherenergy carbon ions. An increase of maximum energies for protons at the thinnest targets and for $\mathrm{CP}$ is also observed; however, the effect is much less pronounced than for carbon ions. The highest $\mathrm{C}^{6+}$ energies observed (for 10-nm targets) are of the order of $25 \mathrm{MeV} / \mathrm{u}(300 \mathrm{MeV})$, to our knowledge the highest carbon ion energies reported so far for tens of femtosecond pulses.

To confirm the scenario suggested by the data, 2D and 3D PIC simulations were carried out employing the ALaDyn code $[35,36]$. The simulation box was a moving window of $51 \mu \mathrm{m}$ along $X$ (the laser-propagation direction) and $100 \mu \mathrm{m}$ wide along both $Y$ and $Z$. In the central region $(51 \times 21.8 \times 21.8 \mu \mathrm{m})$, the cell size is $\Delta x=8.3 \mathrm{~nm}, \Delta y=\Delta z=$ $4 \Delta x$, which is stretched towards the borders to reduce the computational cost. The grid size is $6144 \times 2048 \times 2048$ cells and 100 particles per cell per species were used, accounting for $4.8 \times 10^{9}$ particles. A large number of $2 \mathrm{D}$ simulations (across the $X Y$ plane) were run for two configurations, with the exact same parameters as the 3D runs, but with an increased transverse resolution $\Delta y=\Delta x$, no stretched grid, and 200 instead of 100 macroparticles per cell. The laser-pulse duration (FWHM of the intensity profile) was $\tau=40 \mathrm{fs}$ with a peak intensity
$I=5.5 \times 10^{20} \mathrm{~W} / \mathrm{cm}^{2}$ and a focal spot at waist $w_{0}=3 \mu \mathrm{m}$. The target was composed of two layers. The first layer contained ions with charge-to-mass ratio $Z / A=1 / 2$ (i.e., $\mathrm{C}^{6+}$ ), thickness $\ell_{t}$, and initial electron density $n_{t}=100 n_{c}$. The following layer was a low-density proton layer of thickness $\ell_{r}=12.5 \mathrm{~nm}$ and electron density $n_{r}=10 n_{c}$. This configuration mimics the carbon foil with contaminants on the rear side and allows us to differentiate the dynamics of the different charge states. For computational feasibility, in the simulations the target density and thickness were, respectively, lower and larger than in the experiment while their product, i.e., the areal density, covered the same range employed in the experimental run considering that the estimated electron density for solid carbon is $n_{e} \simeq 350 n_{c}$. For the 3D simulations the areal density was chosen to correspond to the thinnest cases tested in the experiment, whereas in the 2D mode a large parameter scan was carried out considering different target thicknesses.

Figure 4 shows a snapshot of the ion and electron densities obtained from the 3D simulation with $\mathrm{CP}$ and LP. A strong depression of the electron density is observed in the central region at the later time shown, at which time a significant transmission of the laser pulse occurs. The 3D simulation shows how the transition to transparency occurs much faster for LP (between $t \simeq 33 \mathrm{fs}$ and $50 \mathrm{fs}$, which is before the peak of the laser pulse reaches the foil) than for $\mathrm{CP}$, explaining the lower proton energies observed in the former case. The pulse transmission is much stronger for LP than for $\mathrm{CP}$ and may lead to a polarization-dependent transverse shaping of the pulse [37].

For LP, the proton layer is promptly accelerated and detaches from the ion bulk (see Fig. 4). The ion acceleration process is, in this case, essentially due to the sheath field. Using CP the electron heating is strongly suppressed, but even in the CP case, due to the locally non-normal incidence associated to the foil deformation, a small fraction of the electrons escape towards the rear side (see the two lobes of 


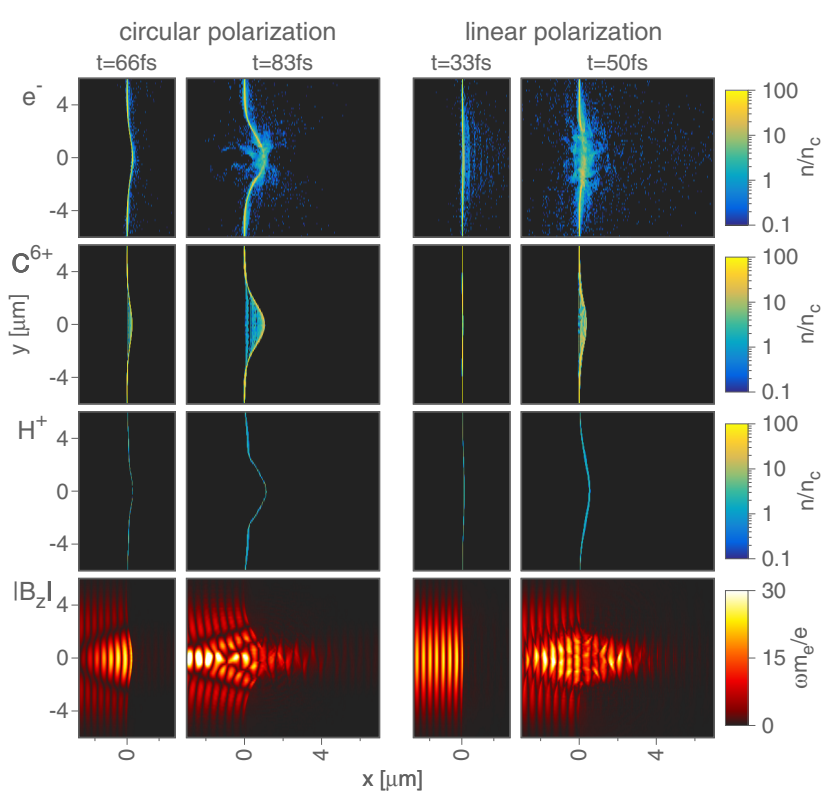

FIG. 4. 3D PIC simulations showing the electron, carbon, and proton densities at different times for both LP and CP. The plot show the density and the magnetic field $B_{z}$ (corresponding to the laser field for LP) in the plane $x-y$, which corresponds to the polarization plane for LP.

electrons above and below the laser axis at $t=83 \mathrm{fs}$ ), building a smaller TNSA field which initially accelerates the protons before RPA becomes the dominant process. The majority of the $\mathrm{C}^{6+}$ ions are bunched and accelerated as a whole by the radiation pressure until the target becomes transparent.

The strong dependences on target thickness and polarization reported in the data of Fig. 2 (and, in particular, the large increase in $\mathrm{C}^{6+}$ energies observed for the thinnest targets employed and CP) suggest that RPA is the dominant acceleration mechanism for the carbon ions. Proton spectra show a similar trend with thickness and polarization, but with less marked differences: at $10 \mathrm{~nm}$, the proton energy for CP is marginally higher than for LP and, while decreasing the target thickness from 100 to $10 \mathrm{~nm}$, proton energies increase by a factor of $\sim 3$ compared to the factor of $\sim 8$ observed for the $\mathrm{C}^{6+}$ ions. Our explanation for the different behavior between the two charged species is related to the onset of significant transmission of the laser pulse through the foil. First, we recall that the threshold condition for the onset of relativistic transparency is equivalent to the condition for which the radiation pressure on electrons equals the maximum possible value of the backholding electrostatic tension exerted by the ions [25]. Thus, the onset of transparency implies that some electrons are swept away from the transmitted laser pulse, creating an electrostatic field at the target rear, which causes the detachment and further acceleration of protons. In contrast, the electron density remains high in correspondence to the carbon layer, stabilizing its acceleration.
Notice that, in principle, the loss of electrons at the rear side may act to further increase transmission through the target and decrease the radiation pressure, so that the RPA action is maintained on the heavier ions (which remain close to the majority of electrons) while the lighter protons get some additional boost from the rear-side electrostatic field.

The strong effect of the onset of pulse transmission in our conditions is also supported by transverse images of the proton beam on RCF; they are similar to those obtained in [38], where their relation with polarization-dependent relativistic transparency is discussed in detail. Overall, our results suggests that transparency effects are the main limiting factor for RPA at present.

The maximum ion and proton energies obtained in the 3D simulations, for 10-nm amorphous carbon targets with similar conditions to this experiment, were $23 \mathrm{MeV} / \mathrm{u}$ $\left(\mathrm{C}^{6+}\right)$ and $34 \mathrm{MeV}$ (protons) for $\mathrm{CP}$ and $15 \mathrm{MeV} / \mathrm{u}$ $\left(\mathrm{C}^{6+}\right)$ and $24 \mathrm{MeV}$ (protons) for LP. The values reproduce the polarization trends observed in the experiment, also displaying a substantial quantitative agreement for the $\mathrm{CP}$ data. Figure 4 also shows the onset of transverse modulations in the density profiles, which may be related to Rayleigh-Taylor instabilities driven by the acceleration of the target $[39,40]$.

The onset of transparency determines the thickness at which the highest ion energy is observed. This has been investigated with a set of parametric 2D simulations for different target thicknesses. As shown in Fig. 5, the optimal thickness is a consequence of the earlier onset of pulse transmission (or target transparency). The thickness dependence is in qualitative agreement with the experiment, as shown in Fig. 3(a). The difference in the ion energies (higher for 2D simulations) may be ascribed to the transition to transparency being slower in $2 \mathrm{D}$ than in the more realistic 3D case, in which the target rarefaction is

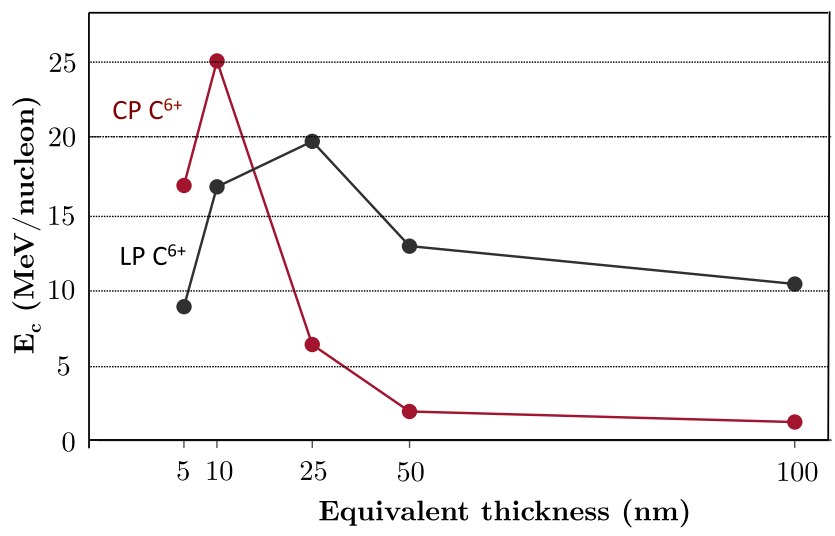

FIG. 5. 2D PIC simulations. Maximum carbon ion energy obtained by varying the thickness of the target for CP and LP. The target thickness is normalized to match the same areal density of solid carbon. 
faster, with the acceleration phase continuing for a longer time in the 2D simulations [39].

In conclusion, in an interaction regime employing ultrashort (50 fs) laser pulses and ultrathin foils $(10-100 \mathrm{~nm}$ carbon), we have observed a strong dependence of the characteristics of the accelerated ions on the target thickness and the laser polarization. The effect is particularly pronounced for carbon ions, for which a "crossover" in the maximum energy for the two different polarizations was evident in both experimental results and supporting PIC simulations. For the thicker targets, the maximum ion energies were higher for LP pulses; below $25 \mathrm{~nm}$, however, the situation changed, producing significantly higher energies for CP laser pulses. This provides evidence that a regime in which RPA is the dominant acceleration mechanism can be accessed at current intensities by careful control of the interaction parameters (pulse contrast, polarization, and target thickness).

Data associated with the results published in this paper can be accessed at [41].

The authors acknowledge funding from the Engineering and Physical Sciences Research Council (Grants No. EP/ K022415/1, No. EP/J003832/1, No. EP/J500094/1, No. EP/I029206/1, No. EP/L002221/1, and No. EP/ J002550/1) and facility access provided by the Science and Technology Facility Council. The simulations were run on 16384 BlueGene/Q cores on FERMI at CINECA (Bologna, Italy). The authors also acknowledge support from the laser and technical staff and target fabrication group at the Rutherford Appleton Laboratory.

* Corresponding author.

d.doria@qub.ac.uk

†Present address: LULI, Sorbonne Universites-UPMC, École Polytechnique, CNRS, CEA, 75005 Paris, France and LESIA, Observatoire de Paris, CNRS, UPMC, UPD, 92195 Meudon, France.

${ }^{\ddagger}$ Corresponding author. m.borghesi@qub.ac.uk

[1] A. Macchi, M. Borghesi, and M. Passoni, Rev. Mod. Phys. 85, 751 (2013).

[2] H. Daido, M. Nishiuchi, and A. S. Pirozhkov, Rep. Prog. Phys. 75, 056401 (2012).

[3] T. E. Cowan et al., Phys. Rev. Lett. 92, 204801 (2004).

[4] M. Schnurer, A. A. Andreev, F. Abicht, J. Branzel, Ch. Koschitzki, K. Yu. Platonov, G. Priebe, and W. Sandner, Phys. Plasmas 20, 103102 (2013).

[5] C. Danson, D. Hillier, N. Hopps, and D. Neely, High Power Laser Sci. Eng. 3, e3 (2015).

[6] M. Roth, T. E. Cowan, M. H. Key, S. P. Hatchett, C. Brown, W. Fountain, J. Johnson, D. M. Pennington, R. A. Snavely, S. C. Wilks, K. Yasuike, H. Ruhl, F. Pegoraro, S. V. Bulanov, E. M. Campbell, M. D. Perry, and H. Powell, Phys. Rev. Lett. 86, 436 (2001).
[7] P. K. Patel, A. J. Mackinnon, M. H. Key, T. E. Cowan, M. E. Foord, M. Allen, D. F. Price, H. Ruhl, P. T. Springer, and R. Stephens, Phys. Rev. Lett. 91, 125004 (2003).

[8] Y. Kitagawa et al., Phys. Rev. Lett. 114, 195002 (2015).

[9] S. V. Bulanov, T. Zh. Esirkepov, V. S. Khoroshkov, A. V. Kuznetsov, and F. Pegoraro, Phys. Lett. A 299, 240 (2002).

[10] V. Malka, S. Fritzler, E. Lefebvre, E. dHumières, R. Ferrand, G. Grillon, C. Albaret, S. Meyroneinc, J. P. Chambaret, A. Antonetti, and D. Hulin, Med. Phys. 31, 1587 (2004).

[11] K. W. Ledingham, P. R. Bolton, N. Shikazono, and C. M. Ma, Appl. Clay Sci. 4, 402 (2014).

[12] S. Kar, A. Green, A. Alejo, H. Ahmed, A. P. L. Robinson, M. Cerchez, R. Clarke, D. Doria, S. Dorkings, J. Fernandez, S. R. Mirfayzi, P. McKenna, K. Naughton, D. Neely, P. Norreys, C. Peth, J. A. Ruiz, J. Swain, O. Willi, and M. Borghesi, New J. Phys. 18, 053002 (2016).

[13] M. Roth et al., Phys. Rev. Lett. 110, 044802 (2013).

[14] K. W. Ledingham, P. McKenna, and R. P. Singhal, Science 300, 1107 (2003).

[15] N. V. Zamfir, Eur. Phys. J. Spec. Top. 223, 1221 (2014).

[16] S. C. Wilks, A. B. Langdon, T. E. Cowan, M. Roth, M. Singh, S. Hatchett, M. H. Key, D. Pennington, A. MacKinnon, and R. A. Snavely, Phys. Plasmas 8, 542 (2001).

[17] H. Powell, M. King, R. J. Gray, D. A. MacLellan, B. Gonzalez-Izquierdo, L. C. Stockhausen, G. Hicks, N. P. Dover, D. R. Rusby, D. C. Carroll, H. Padda, R. Torres, S. Kar, R. J. Clarke, I. O. Musgrave, Z. Najmudin, M. Borghesi, D. Neely, and P. McKenna, New J. Phys. 17, 103033 (2015).

[18] T. Ceccotti, A. Lévy, H. Popescu, F. Réau, P. D’Oliveira, P. Monot, J. P. Geindre, E. Lefebvre, and Ph. Martin, Phys. Rev. Lett. 99, 185002 (2007).

[19] D. Neely, P. Foster, A. Robinson, F. Lindau, O. Lundh, A. Persson, C.-G. Wahlstrom, and P. McKenna, Appl. Phys. Lett. 89, 021502 (2006).

[20] P. McKenna, F. Lindau, O. Lundh, D. Neely, A. Persson, and C.-G. Wahlström, Phil. Trans. R. Soc. A 364, 711 (2006).

[21] T. Esirkepov, M. Borghesi, S. V. Bulanov, G. Mourou, and T. Tajima, Phys. Rev. Lett. 92, 175003 (2004).

[22] A. P. L. Robinson, M. Zepf, S. Kar, R. G. Evans, and C. Bellei, New J. Phys. 10, 013021 (2008).

[23] X. Zhang, B. Shen, X. Li, Z. Jin, and F. Wang, Phys. Plasmas 14, 073101 (2007).

[24] O. J. Klimo, J. Psikal, J. Limpouch, and V. T. Tikhonchuk, Phys. Rev. ST Accel. Beams 11, 031301 (2008).

[25] A. Macchi, S. Veghini, and F. Pegoraro, Phys. Rev. Lett. 103, 085003 (2009).

[26] B. Qiao, M. Zepf, M. Borghesi, and M. Geissler, Phys. Rev. Lett. 102, 145002 (2009).

[27] F. Dollar, C. Zulick, A. G. R. Thomas, V. Chvykov, J. Davis, G. Kalinchenko, T. Matsuoka, C. McGuffey, G. M. Petrov, L. Willingale, V. Yanovsky, A. Maksimchuk, and K. Krushelnick, Phys. Rev. Lett. 108, 175005 (2012).

[28] A. Henig, S. Steinke, M. Schnürer, T. Sokollik, R. Hörlein, D. Kiefer, D. Jung, J. Schreiber, B. M. Hegelich, X. Q. Yan, J. Meyer-ter-Vehn, T. Tajima, P. V. Nickles, W. Sandner, and D. Habs, Phys. Rev. Lett. 103, 245003 (2009).

[29] S. Steinke, P. Hilz, M. Schnurer, G. Priebe, J. Branzel, F. Abicht, D. Kiefer, C. Kreuzer, T. Ostermayr, J. Schreiber, 
A. A. Andreev, T. P. Yu, A. Pukhov, and W. Sandner, Phys. Rev. ST Accel. Beams 16, 011303 (2013).

[30] J. H. Bin, W. J. Ma, H. Y. Wang, M. J. V. Streeter, C. Kreuzer, D. Kiefer, M. Yeung, S. Cousens, P. S. Foster, B. Dromey, X. Q. Yan, R. Ramis, J. Meyer-ter-Vehn, M. Zepf, and J. Schreiber, Phys. Rev. Lett. 115, 064801 (2015).

[31] S. Kar, K. F. Kakolee, B. Qiao, A. Macchi, M. Cerchez, D. Doria, M. Geissler, P. McKenna, D. Neely, J. Osterholz, R. Prasad, K. Quinn, B. Ramakrishna, G. Sarri, O. Willi, X. Y. Yuan, M. Zepf, and M. Borghesi, Phys. Rev. Lett. 109, 185006 (2012).

[32] B. Dromey, S. Kar, M. Zepf, and P. Foster, Rev. Sci. Instrum. 75, 645 (2004).

[33] D. Doria, S. Kar, H. Ahmed, A. Alejo, J. Fernandez, M. Cerchez, R. J. Gray, F. Hanton, D. A. MacLellan, P. McKenna, Z. Najmudin, D. Neely, L. Romagnani, J. A. Ruiz, G. Sarri, C. Scullion, M. Streeter, M. Swantusch, O. Willi, M. Zepf, and M. Borghesi, Rev. Sci. Instrum. 86, 123302 (2015).

[34] A. Mančić, J. Fuchs, P. Antici, S. A. Gaillard, and P. Audebert, Rev. Sci. Instrum. 79, 073301 (2008).
[35] C. Benedetti, A. Sgattoni, G. Turchetti, and P. Londrillo, IEEE Trans. Plasma Sci. 36, 1790 (2008).

[36] P. Londrillo, C. Benedetti, A. Sgattoni, and G. Turchetti, Nucl. Instrum. Methods Phys. Res., Sect. A 620, 28 (2010).

[37] B. Gonzalez-Izquierdo, R. J. Gray, M. King, R. J. Dance, R. Wilson, J. McCreadie, N. M. H. Butler, R. Capdessus, S. Hawkes, J. S. Green, M. Borghesi, D. Neely, and P. McKenna, Nat. Phys. 12, 505 (2016).

[38] B. Gonzalez-Izquierdo, M. King, R. J. Gray, R. Wilson, R. J. Dance, H. Powell, D. A. Maclellan, J. McCreadie, N. M. H. Butler, S. Hawkes, J. S. Green, C. D. Murphy, L. C. Stockhausen, D. C. Carroll, N. Booth, G. G. Scott, M. Borghesi, D. Neely, and P. McKenna, Nat. Commun. 7, 12891 (2016).

[39] A. Sgattoni, S. Sinigardi, and A. Macchi, Appl. Phys. Lett. 105, 084105 (2014).

[40] F. Pegoraro and S. V. Bulanov, Phys. Rev. Lett. 99, 065002 (2007).

[41] DOI: 10.17034/3c451ca4-7a2a-489e-b449-e0bdb83ffa85. 